\title{
El papel de la World Wide Web y la telefonía móvil en las relaciones sociales de los jóvenes de origen latinoamericano en Cataluña
}

\author{
Amparo Huertas Bailén ${ }^{1}$
}

El objetivo de este texto es indagar sobre la forma en que la World Wide Web y la telefonía móvil influyen en el establecimiento de las relaciones sociales de los adolescentes (entre 15 y 19 años) en un contexto multicultural a partir de un estudio empírico de carácter cualitativo realizado el 2009. En esta investigación ha participado un total de 85 estudiantes (75 de origen latinoamericano y 10 autóctonos) residentes en L'Hospitalet de Llobregat, una localidad cercana a Barcelona cuya población inmigrada supera el $25 \%$. Las principales conclusiones señalan que la tendencia dominante es hacia las relaciones endógenas (autóctonos con autóctonos y migrantes con migrantes) y, en consecuencia, el uso de estas tecnologías de la comunicación también se encamina en este sentido.

Palabras claves: tecnologías de la comunicación, adolescencia, migración.

The role of the World Wide Web and mobile telephony in the social relationships among Latin American adolescent in Catalonia. This article tries to think about the influence of the World Wide Web and mobile telephony on the relationships among adolescents (15-19 years old) in a multicultural context on the basis of an empiric qualitative study carried out during 2009. 85 students participated actively in this investigation ( 75 from Latin America and 10 Spanish ones). They all live in L'Hospitalet de Llobregat, a city near Barcelona where more than $25 \%$ of the population is made up of immigrants. The main conclusion indicates that the natural tendency among young people is to find friends with similar characteristics (natives with natives and immigrants with immigrants), so that the use of those communication technologies goes in the same direction.

Keywords: communication technologies, adolescence, migration.
O objetivo deste trabalho é investigar o modo como a World Wide Web e a telefonia móvel influenciam o estabelecimento das relaçôes sociais dos adolescentes (entre 15 e 19 anos) em um contexto multicultural a partir de um estudo empirico qualitativo realizado em 2009. Esta pesquisa envolveu um total de 85 alunos (75 da América Latina e 10 espanhóis) residentes em L'Hospitalet de Llobregat, uma cidade perto de Barcelona, onde a população imigrante é superior a 25\%. As principais conclusões indicam que a tendência dominante entre os jovens são as relaçôes endógenas (nativos com nativos e migrantes com migrantes) e, consequentemente, o uso dessas tecnologias de comunicação também se encaminham nesta direção.

Palavras-chaves: tecnologias da comunicação, adolescência, migração.

\footnotetext{
${ }^{1}$ Instituto de la Comunicación de la Universidad Autónoma de Barcelona (InCom-UAB). Departamento de Comunicación Audiovisual y de Publicidad I. Edificio N. Campus UAB. 08193 Cerdanyola del Vallés, Barcelona, España. E-mail: amparo.huertas@uab.cat.
} 


\section{Introducción}

Las tecnologías de la comunicación juegan hoy en día un papel esencial en las relaciones sociales (colectivas y personales) de los adolescentes. En Cataluña, contexto en el que se ha realizado el trabajo de campo cuyos resultados aquí se presentan, el $87 \%$ de los jóvenes (de 15 a 19 años) tiene teléfono móvil y el $91 \%$ de estos usuarios lo emplea para enviar mensajes de texto. En lo referente a la World Wide Web, el 91\% de los adolescentes residentes en esta Comunidad Autónoma de España accede regularmente a la red global y más del $90 \%$ de estos internautas utiliza el correo electrónico y el servicio de mensajería instantánea. Son datos del Barómetro de la Comunicación y la Cultura ${ }^{2}$ referentes al período comprendido entre marzo de 2008 y febrero de 2009. Ante estas cifras, no cabe ninguna duda de que gran parte de las relaciones sociales de estos jóvenes son de carácter mediado (comunicación mediada) y tampoco es de extrañar que ya existan conceptos como e-generation o net-generation para designar de manera específica a esta generación.

Desde el ámbito académico, el interés por estudiar el impacto social de la red global surgió casi en el mismo momento en que ésta pasó a ser accesible a la ciudadanía. Tras revisar las principales aportaciones realizadas sobre la World Wide Web, Scolari (2008) las clasifica del siguiente modo: los sociólogos se interesaron rápidamente por el estudio de las "comunidades virtuales" abordándolas a partir de la noción de "redes sociales"; los antropólogos indagaron sobre la cibernética, las intersecciones entre individuos, sociedad y redes digitales; la etnografía trató básicamente las cuestiones relacionadas con la identidad $y$, por último, los lingüistas analizaron su influencia en la evolución del habla. No obstante, Scolari (2008) indica el 2004 como año clave en este reconocimiento del impacto social de la red global como objeto de estudio, ya que es en esa fecha cuando se crea la Asociación de los Investigadores de Internet. En cambio, alrededor del sector de la telefonía móvil, lo que ha proliferado más son los estudios de carácter comercial, razón por la cual su investigación desde la perspectiva académica todavía se halla en fase de iniciación (Aguado y Martínez,2006). No obstante, el trasvase de los medios de comunicación hacia el móvil como soporte de recepción, unido a la ampliación de sus usos potenciales, está cambiando la situación. Una prueba de ello son los estudios sobre "audiencia móvil", siendo uno de los pioneros el trabajo de Castells (2006).

La investigación que aquí se presenta se enmarca en el área de la Comunicación. El objetivo es indagar sobre la forma en que la World Wide Web y la telefonía móvil influyen en la configuración de las redes sociales de los adolescentes (de 15 a 19 años) en un entorno multicultural y, más concretamente, en su repercusión en los procesos de sociabilidad entre latinoamericanos y autóctonos residentes en Cataluña. Si bien es cierto que la tendencia es hacia un aumento de la conectividad a Internet vía telefonía móvil (Igarza, 2009), en el momento de realizar el trabajo de campo que aquí se presenta éstos son dos entornos tecnológicos que todavía actúan mayoritariamente de forma independiente: mientras que el móvil se enmarca básicamente en las relaciones con personas que viven en el mismo país, la red global incide, además, en las de carácter transnacional. Este estudio forma parte de un proyecto más amplio titulado Cohesión social y Juventud. Consumo mediático y prácticas culturales de los jóvenes -migrantes y autóctonos- en Cataluña, realizado en el marco del Instituto de la Comunicación de la UAB (InCom-UAB) con la ayuda económica del Departament de Cultura i Mitjans de Comunicació de la Generalitat de Catalunya ${ }^{3}$.

\section{Definiendo los "procesos socio-afectivos-tecnológicos"}

Los tres ejes temáticos que conforman el marco teórico de esta investigación son (a) la etapa vital de la adolescencia, (b) la influencia de las tecnologías de la comunicación en la definición de las relaciones sociales y (c) el proceso de integración socio-cultural de la población de origen extranjero.

Para el estudio de la población juvenil migrante, lo primero que hay que tener en cuenta es en qué consiste la adolescencia. Recogemos aquí la definición de Livingstone

\footnotetext{
${ }^{2}$ Éste es un estudio estadístico, elaborado por FUNDACC, que ofrece periódicamente información sobre el consumo mediático y cultural de la población residente en Cataluña.

${ }^{3}$ El equipo de investigación, dirigido por la autora de este artículo, estuvo formado por Adriana Ibiti, Núria Reguero Jiménez y Luis Felipe Velásquez Ugalde. El trabajo se hizo bajo la supervisión del catedrático Miquel de Moragas i Spà.
} 
(1998). En un trabajo sobre el consumo mediático de la población adolescente europea, esta autora definió esta etapa de la vida como ese período en que se hace la transición desde el entorno familiar a la cultura juvenil. Se trata de un camino difícil, ya que está marcado por unos rasgos psicológicos que lo dificultan: la adolescencia es un período en el que se acentúa la vulnerabilidad de la personalidad, se admira irrevocablemente "lo alternativo" y se desea una rápida desvinculación de la familia, según John Coleman (1985), fundador de TSA (Trust for the Study of Adolescence).Pero todavía resulta más complicado cuando coincide con un proceso de integración en un entorno cultural ajeno. El joven migrante, como cualquier adolescente, se encuentra con la necesidad de formar su propia identidad y de ser aceptado por los otros; y, al mismo tiempo, ha de adaptarse a un contexto en el que sus raíces culturales pueden ser peyorativamente consideradas como minoritarias. Prada (2005), a partir de un estudio llevado a cabo en España, apunta que lo más habitual es que se dé una contraposición entre la "cultura de la calle" (el "buscarse la vida") y la "cultura universitaria" (la "excelencia del saber") y, por su parte, Laura Zanfrini (2007) indica la alta probabilidad de que aparezca lo que se llama "etnicidad reactiva”. Ésta consiste en el deseo de conocer y preservar las raíces culturales propias e, incluso, de llegar a ser reconocido de manera casi exclusiva por ellas, una necesidad muy frecuente sobre todo en las "segundas generaciones".

Pero tan importante es la actitud del joven migrante como la del joven autóctono en el establecimiento de las relaciones sociales. Así, Zanfrini (2007) también apunta las nociones de "discriminación directa", la que aparece cuando el autóctono no trata bien al migrante (sobre todo como consecuencia de una actitud racista y discriminatoria basada en el género, la clase social y/o la religión del otro), y de "identidad asignada" (cuando el autóctono asigna al migrante un perfil psicológico en base a su nacionalidad o aspecto físico, lo que actúa en beneficio del fortalecimiento de los estereotipos). De hecho, Prada (2005) señala también que el adolescente autóctono suele describir al extranjero como una persona "atrasada" y observar el proceso de integración como aquel que "le obliga a ser amigo de los 'otros".

Para tratar acerca de la influencia de las tecnologías de la comunicación en la definición de las relaciones sociales, partimos de las reflexiones del filósofo
Pierre Lévy (2007). "La distinción entre cultura (la dinámica de las representaciones), sociedad (la gente, sus lazos, sus intercambios, sus relaciones de fuerza) y técnica (los artefactos eficaces) no puede ser más que conceptual" (Lévy, 2007, p. 7), ya que estos tres conceptos, cultura, sociedad y técnica, son inexorablemente dependientes entre si. Lévy defiende de este modo la noción de "procesos socio-culturalestecnológicos", un concepto que recogemos aquí aunque, debido a la delicada etapa de la adolescencia, haciendo más énfasis en lo afectivo que en lo cultural, por lo que podríamos hablar del estudio de "procesos socioafectivos-tecnológicos".

Por último, las aportaciones teóricas sobre integración socio-cultural que se asumen en este marco teórico están datadas mayoritariamente en las últimas dos décadas, aunque también se ha hecho un repaso histórico desde las primeras reflexiones aparecidas en la década de los años 50 en Estados Unidos en respuesta a las particulares circunstancias de aquel país ${ }^{4}$ Seleccionamos a continuación aquellas que más huella han dejado desde el mismo inicio de este trabajo, teniendo en cuenta que todas ellas convergen en una misma idea: los procesos de socialización son complejos $\mathrm{y}$ heterogéneos.

Will Kymlicka (1996), especializado en Filosofia y Política Pública, plantea que el proceso de integración social supone al migrante un coste muy elevado, el volumen del cual depende de la duración de ese proceso -más largo para las culturas no liberales en contextos liberales-, la edad de la persona en el momento de llegar y las similitudes entre las lenguas e historias que entran en convivencia. En segundo lugar, de entre las interesantes aportaciones del antropólogo Ulf Hannerz (1998), dado que nuestro objeto de estudio se centra en las relaciones sociales, destaca especialmente su advertencia de que, en los contextos multiculturales, se dan diferentes grados de familiaridad y de conocimiento mutuo, así como de intenciones de conocer culturas y prácticas ajenas. Y, en tercer lugar, también conviene apuntar la idea de que los contextos sociales (de salida y de llegada) de los migrantes, sus circunstancias y expectativas personales son muy diversos (Cogo et al., 2008). A pesar de que cuando la emigración se produce en edad infantil o juvenil ésta suele

\footnotetext{
${ }^{4}$ Una aportación de enorme interés es la de Robert K. Merton (1957), quien en aquel momento ya apuntó las paradojas de la integración. A modo de ejemplo, recogemos aquí una de sus ideas: aunque los hijos de los migrantes pueden haber cursado estudios en el nuevo país de residencia, en muchos casos no llegan a tener las mismas oportunidades de movilidad social que los autóctonos.
} 
ser el resultado de una decisión familiar (normalmente de los progenitores), las circunstancias son también muy variadas en estos casos. A partir de las investigaciones realizadas desde el InCom-UAB, hemos podido comprobar la diversidad de situaciones que pueden darse. Sirvan a título de ejemplo los siguientes casos: jóvenes que emigran únicamente con uno de sus progenitores y no sólo han de aprender a convivir en una nueva ciudad sino que también han de aceptar las nuevas relaciones de pareja que el padre o la madre intentan establecer; niños que han de vivir con familiares que no son sus padres; adolescentes que se ven obligados a viajar para recibir un tratamiento médico o que, después de haber pasado la infancia en su país de nacimiento, se reencuentran con unos padres que les resultan personas desconocidas tanto psicológicamente como físicamente.

\section{Apuntes metodológicos}

Los resultados y conclusiones que se aportan en este texto surgen de un trabajo de campo cualitativo realizado entre marzo y junio de 2009 en L'Hospitalet de Llobregat, una población situada en el extrarradio de Barcelona con más del 25\% de población extranjera (mayoritariamente de origen latinoamericano). En concreto, el trabajo se ha llevado a cabo en dos centros escolares de dicho municipio, tras ser aceptada nuestra propuesta por los respectivos equipos directivos. La metodología se ha desarrollado en tres fases y se ha centrado en tres variables de análisis: el género, la edad y el tiempo de permanencia en Cataluña.

En un primer momento, se realizó un cuestionario anónimo sobre una muestra formada por el total de estudiantes latinoamericanos matriculados en dichos centros con una edad comprendida entre los 15 y los 19 años. En total, participaron 75 alumnos. En consecuencia, el perfil sociodemográfico de esta muestra estuvo determinado por las características de dicho alumnado. Respecto al país de origen, la mayoría procedía de Ecuador (29) y Bolivia (17); y los otros países presentes en la investigación fueron República Dominicana (8), Colombia (7), Argentina (6), Perú (6) y Honduras (2) (ver Tabla 1).

El objetivo de este cuestionario anónimo era obtener información sobre sus actividades preferidas y sus relaciones sociales con otros jóvenes en el tiempo libre. Se asumía un enfoque muy abierto, ya que una de
Tabla 1. Composición de la muestra que complementó el cuestionario anónimo inicial.

Table 1. Composition of the sample that complemented the initial anonymous questionnaire.

\begin{tabular}{|l|c|}
\hline \multicolumn{2}{|c|}{ Género } \\
\hline Chicos & 27 \\
\hline Chicas & 48 \\
\hline \multicolumn{2}{|c|}{ Edad (años) } \\
\hline 15 & 11 \\
\hline 16 & 23 \\
\hline 17 & 23 \\
\hline 18 & 11 \\
\hline 19 & 7 \\
\hline \multicolumn{2}{|c|}{ Tiempo de permanencia en Cataluña (años) } \\
\hline Nivel 1 (0-2,5) & 17 \\
\hline Nivel 2 (2,5-3,5) & 12 \\
\hline Nivel 3 (3,5-5) & 13 \\
\hline Nivel 4 (5-7) & 21 \\
\hline Nivel 5 (más de 7) & 12 \\
\hline TOTAL de jóvenes latinoamericanos consultados & 75 \\
\hline
\end{tabular}

Fuente: Elaboración propia a partir del trabajo de campo realizado en L'Hospitalet de Llobregat (Barcelona-Cataluña-España).

las intenciones era detectar si los vínculos vía Internet o telefonía móvil surgían espontáneamente en su discurso.

Una vez analizada la información recogida en estos cuestionarios, se comprobó que Internet sólo aparecía mencionada por 29 de los 75 jóvenes consultados. De este modo, aunque ya estaba planificada una segunda fase, se puso en evidencia que ésta era realmente imprescindible para poder abordar la cuestión de la telefonía móvil. En esta segunda etapa se llevaron a cabo 5 focus groups y, en total, participaron 32 jóvenes (22 de origen latinoamericano -que ya habían participado en la fase inicial- y 10 autóctonos; 19 eran mujeres y 13 eran hombres).

La principal finalidad de estos focus groups era tener una visión más completa de las relaciones sociales entre autóctonos y migrantes. Se crearon diferentes contextos comunicativos, buscando siempre en la medida de lo posible un equilibrio por género e intentando que estuviera presente el mayor rango de edades entre los 15 y los 19 años en cada focus group. Finalmente, una agrupación reunió a seis estudiantes latinoamericanos; otra, a siete autóctonos; una tercera 
fue mixta (tres latinoamericanos y tres autóctonos); la cuarta estuvo formada por seis latinoamericanos con más de seis años de permanencia en Cataluña y la última, por siete que tan sólo llevaban dos años en esta Comunidad Autónoma.

Un aspecto que sorprendió al equipo que realizó el trabajo de campo ${ }^{5}$ es que los comentarios del focus group formado exclusivamente por autóctonos fueron en algunos momentos algo agresivos; por contra, en presencia de latinoamericanos, mostraron un lenguaje más comedido. De hecho, en el marco global de esta investigación, se detectó claramente que la actitud del autóctono es mayoritariamente negativa, con matices racistas y xenófobos, cuando se expresa sin la presencia del migrante (éstas fueran algunas de las ideas recogidas: "Los migrantes tienen mejor trato por parte de los servicios públicos sólo porque son migrantes", "vienen a quitarnos el trabajo" o "ahora en las calles y en el colegio hay más inseguridad por su culpa").

La última etapa consistió en la realización de 9 entrevistas en profundidad a jóvenes latinoamericanos, la mayoría se hizo a chicas (6 frente a 3). Este desequilibrio por géneros se debe a que se seleccionaron a alumnos que en las fases anteriores habían mostrado rasgos peculiares de interés para nuestro objeto de estudio o bien que habían tenido problemas a la hora de expresarse. Además, hay que tener en cuenta que el alumnado de origen latinoamericano de los centros escolares donde se hizo el trabajo de campo era mayoritariamente femenino (algunos profesores argumentaron que esto podía ser debido a que ellos prefieren abandonar los estudios e incorporarse al mundo laboral antes de finalizar el período escolar). El propósito de estas conversaciones guiadas era ahondar en los aspectos abordados en las etapas anteriores, pero desde una perspectiva más personal, atendiendo a las experiencias vividas a título individual.

A lo largo del texto se reproducen literalmente -sin corregir las faltas ortográficas- citas extraídas de los discursos orales y escritos de los estudiantes que han participado en este trabajo. Todas ellas aparecen identificadas indicando el sexo, el país de origen, la edad y, entre paréntesis, el tiempo de permanencia en Cataluña expresado en años cuando se hace referencia a un joven de origen extranjero.

\footnotetext{
${ }^{5}$ Algunos de ellos eran también latinoamericanos.
}

\section{Dominio de las relaciones sociales endógenas}

Los aspectos que más valoran los adolescentes a la hora de hacer amistades son la fidelidad, el optimismo y la sinceridad. Es decir, nociones como la nacionalidad, la etnia o la religión no forman parte de sus discursos cuando explican con quién les gusta pasar su tiempo de ocio. Sin embargo, cuando se estudia el grado real de multiculturalidad de sus grupos de amistades, resulta todo lo contrario. Los autóctonos tienden a relacionarse básicamente con otros autóctonos y los migrantes también se reúnen mayoritariamente con jóvenes con las mismas raíces culturales, dominando así las relaciones endógenas. Ambos colectivos prefieren estar con las personas que les resultan más afines y ambos tienen estereotipos sobre el 'otro' que dificultan la aproximación.

Porque cuando ves alguien de tu país, puedes preguntar de dónde eres y empezar a hablar sobre muchas cosas... (Chico, Ecuador, 17 (2) años).

[sobre los catalanes] Se creen lo que no son. Algunos pasan y no saludan y sacan los latinos aparte (Chico, Ecuador, 16 (2) años).

Hacer amigos españoles me cuesta un poco porque soy muy reservada. Los españoles son más abiertos, más liberales que nosotros (Chica, Ecuador, 15 (2) años).

Yo les pregunto [a los migrantes] por sus cosas. Pero es dificil hablar con ellos porque usan palabras que no entiendo como 'lambón' que quiere decir 'chupaculos' (Chica, España, 15 años).

No cuento mucho de mis cosas a los españoles tampoco lo hacen conmigo, son muy cerrados y no me dan confianza [...] entre los suramericanos nos entendemos mejor (Chica, República Dominicana, 15 (6) años).

[sobre los migrantes] no tienen educación, traen diferentes culturas y jeso qué tiene de bueno? (Chico, Cataluña, 17 años). 
Como resultado de este fenómeno, el centro escolar se convierte en el principal entorno de convivencia multicultural de estos jóvenes. Es más, parece que la relación con jóvenes de otras culturas tiende a centrarse en el desarrollo de las clases y a diluirse en las horas de recreo ${ }^{6}$.

Noté mucho cambio, diferencia entre culturas. No estoy acostumbrado a la manera de ser de los españoles, ni a sus palabras [...] en el colegio se forman grupos de españoles con españoles y luego siguen en el patio (Chico, Argentina, 18 (4) años).

Además, también se ha detectado la tendencia de algunos migrantes a pasar su tiempo libre en espacios donde se potencia únicamente la relación con personas de las mismas raíces culturales. En el caso latinoamericano, las discotecas de música latina son un claro ejemplo, aunque sobre todo destaca como una característica particular de este colectivo la preferencia por organizar/asistir a fiestas particulares en casas de amigos.

[Voya] una [discoteca] que se llama 'Canela', que es de música latina: salsa, bachata, reggaeton... también un poco de house. [La gente que va es] Latina! La mayoría latina... españoles de casualidad, uno o dos... También voy a 'Copacabana'... a bailar, hay mucha gente latina (Chica, Bolivia, 17 (5) años).

Porque en las casas también hay mucha gente que no conoces, es un lugar en que te sientes más tranquila, y sabes que hay gente que conocen los demás, y puedes conocer a amigos de amigos, en cambio en la disco hay mucho ruido para hablar y no hay mucha confianza para hablar (Chica, Ecuador, 15 (6,5) años).

Pero el problema no es sólo entre migrantes y autóctonos. Dentro del colectivo de origen extranjero, también se producen conflictos, incluso entre personas con raíces culturales próximas. Por ejemplo, en este trabajo, se detectaron relaciones difíciles entre dominicanos y ecuatorianos. Ahora bien, el acercamiento más complejo es el de jóvenes de culturas liberales con otros de culturas menos abiertas. Así, por ejemplo, la mayoría de comentarios en este sentido hicieron referencia a conflictos en las relaciones entre latinoamericanos y magrebíes (jóvenes procedentes del norte de África).

Los marroquies no nos gustan, utilizan a las mujeres como un objeto (Chico, Argentina, 18 (4) años).

Cualquiera [podría ser su pareja] bueno, pero no todos... los chicos pakistanies como que no me llegan mucho y los africanos pues tampoco... sobre todo por eso de las mujeres... que no las dejan salir y eso (Chica, Ecuador, 15 (6) años).

Para acabar, no puede obviarse el tema de las bandas juveniles de comportamiento violento. Éste es un asunto que preocupa claramente a padres y educadores, pero también a los propios jóvenes. Muchos de los estudiantes participantes de este proyecto alertaron sobre los problemas que genera relacionarse con este tipo de grupos (según sus propios discursos, incitan a la participación en actos violentos y robos), pero, al mismo tiempo, eran capaces de dar información muy precisa sobre cuestiones como los ritos de entrada y de salida. Todo ello no hizo otra cosa que poner en evidencia que muchos de los miembros de la muestra consultada mantienen contacto con estas agrupaciones, y no sólo los de origen extranjero ${ }^{7}$.

Prefiero estar solo que mal acompañado. Ellos te quieren meter en bandas... tengo amigos que pertenecen a ellas y van a robar (Chico, España, 17 años).

Tenía una amiga por 'el mal camino' y me traía problemas... hasta que me di cuenta que me perjudicaba y la dejé (Chica, Bolivia, 16 (7) años).

Muchas veces la violencia es de latinos con latinos, por ejemplo al mirarse entre ellos por la calle, sobre todo a las chicas de algún chico de una banda [...] Los Latin

\footnotetext{
${ }^{6}$ En uno de los institutos donde se realizó el trabajo de campo se programan actividades deportivas para favorecer la relación entre migrantes y autóctonos en el horario de recreo.

${ }^{7}$ En los dos institutos donde se hizo el trabajo de campo, los estudiantes tienen prohibido llevar indumentaria que les identifique con bandas de este tipo. En uno de ellos, la norma se aplica sólo en los espacios cerrados (en el patio sí pueden utilizar este tipo de ropa) y, en el otro, en todo el recinto.
} 
Kings en clase son tranquilos, antes habia muchos problemas fuera del cole... pero ahora cuando salimos siempre está la policía (Chico, Ecuador, 16 (4) años).

\section{Hacia la comunicación multimedial y participativa}

La información recogida demuestra, sin lugar a dudas, que el ordenador y el teléfono móvil son dos aparatos imprescindibles en la vida cotidiana de los jóvenes. Ahora bien, la utilidad dada a ambos equipos es muy distinta.

En términos generales, el ordenador aparece como un equipo multiusos que muchas veces se activan en simultaneidad. Esta herramienta, además de permitir mantener contactos con otras personas, también se asocia con un fácil acceso a productos mediáticos y culturales (como música, películas o programas de televisión), con la búsqueda de información (principalmente relacionada con tareas escolares) y, de manera más puntual, con el desarrollo de actividades creativas (por ejemplo, diseñar páginas webs o crear documentos power point).

[Me gusta] Estar en el ordenador porq' me entero de cosas y me ayuda en los deberes (Chico, Ecuador, 17 $(1,5)$ años).

Me gusta entretenerme viendo en Internet o en documentales lugares en todo el mundo donde me gustaría viajar en compañía de mis amigos o en expediciones para ver el comportamiento de los animales (Cbico, Colombia, 16 (3) años).

Estar en Internet, conectarme al Messenger, ver mi correo y enviar mails. También ver videos en youtube - alguna película, o también series y telenovelas. Buscar información para algún trabajo de clase (Chica, Perú, 18 (10) años). ${ }^{8}$

Por su parte, el teléfono móvil, además de servir para entablar comunicación con otras personas, aparece muy estrechamente ligado a la seguridad personal. La mayor parte de los jóvenes consultados comentaron que sus padres, de alguna manera, les obligan a llevar un móvil cuando salen de casa con el fin de estar localizables en todo momento y, al mismo tiempo, para poder pedir ayuda en caso de necesidad.

A mi me gusta, yo no me imagino sin él. Mi madre igual me dice que lo lleve, asi puedo avisar si llego tarde y esas cosas (Chica, Honduras, 16 (2) años).

Si, cuando voy de excursión o si voy a una acampada o algo así, siempre tengo que llevarlo (el móvil) y para localizarme (Chico, Ecuador, 17 (3) años).

Si, a mi mamá... A mi hermano, nunca le llamo [Risas] $Y$ si le llamo es porque no tengo llaves o no sé donde están... (Chica, Bolivia, 16 (6) años).

Si se compara el papel de la WWW con el de la telefonía móvil en el ámbito de las relaciones sociales de los jóvenes, se desprende que la importancia de la red global es mucho mayor. No es que al joven no le guste utilizar el móvil. Según el trabajo de campo, les gusta hablar por teléfono (algunos apuntan incluso "sentirse más importantes” cuando recibían una llamada), pero, por una cuestión económica, han de optar por los mensajes cortos y una comunicación textual de este tipo acaba resultando más atractiva en la World Wide Web, donde la respuesta puede ser inmediata e, incluso, compartida por diferentes personas en tiempo real. Además, la red permite un envío más fácil de fotografías o vídeos (comunicación multimedial) y ofrece una posibilidad de relación que tiene muy buena acogida entre los jóvenes, los videojuegos online (comunicación participativa).

Pasar la tarde hablando con los amigos/as por Internet, jugar con ellos... me gusta bastante los ordenadores e Internet i jugar con los amigos en red mientras hablo con ellos, en vez de estar hechando la siesta hago eso que es como si fuera mi siesta porque asi me siento bien (Cuestionario 105, chica de España de 19 años).

[Me gusta] jugar al ordenador, por que es muy divertido y puedes jugar partidas online con amigos de

${ }^{8}$ Traducción del catalán original. 
clase, o que juegan al mismo juego (Chico, Ecuador, 15(6) años).9

De los 75 estudiantes que complementaron el cuestionario inicial, 29 (38\%) apuntaron utilizar la red para mantener y fortalecer sus relaciones sociales, sobre todo mediante el uso del MSN (Messenger). Por el contrario, la mención del e-correo fue muy puntual.

Comunicarse con amigos a través del ordenador suele estar unido a momentos de ocio en casa, pero parece ser que ésta es una práctica más habitual entre las chicas que entre los chicos. Esta actividad aparece en muchas ocasiones como la preferida por las chicas (a la misma altura que "estar con los amigos" o "escuchar música"). En cambio, entre los chicos, sólo destaca como segunda o tercera opción para ocupar el tiempo libre y, además, aparece entre las preferencias minoritarias. Este dato, unido a otros aspectos (como el hecho de que prácticamente sólo fueron chicas las que indicaran "descansar en casa", "estar con la familia" o "mirar la televisión" como actividades de ocio favoritas), parece revelar que ellas pasan más tiempo en el hogar, lo que, de alguna manera, desemboca en que el ordenador tenga un papel más destacado en el establecimiento de sus relaciones.

Por edades, las relaciones sociales vía internet parecen concentrarse entre los 17 y 18 años. La información obtenida no explica por qué el consumo no destaca a los 15 o 16 años, pero sí da cuenta de que este servicio suele deslumbrar cuando se descubre para pasar después a ser una actividad aburrida y ello puede justificar que aparezca concentrado en un período temporal muy concreto.

Antes me gustaba estar en el Messenger, enganchada... Y ahora ya no. Ahora, a veces, me pongo, pero, a jugar las cartas esas que tiene y ya está [...] Es que no me llama ya la atención. Antes es que ni sé de lo que hablábamos... [Risas] Ahora no entiendo por qué me llamaba tanto la atención porque abi bablando y me aburro y al final, nada, me voy (Chica, Argentina, 19 (6) años).

Pero me aburro chateando por el MSN y prefiero quedar en persona. Por el chat aunque esté hablando se termina la conversación y ya no sé qué decir (Chica, Ecuador, 15 (6,5) años).

\footnotetext{
${ }^{9}$ Traducción del catalán original.
}

En lo que se refiere a la variable del tiempo de permanencia en Cataluña, las relaciones mediadas vía WWW parecen concentrarse durante los primeros años después de la llegada (0-2,5 años). A medida que pasa el tiempo, las actividades deportivas y las salidas con los amigos van ganando peso. Todo lo contrario sucede con la "audición de música", la actividad más estable desde el punto de vista de esta variable.

De las 29 personas que apuntaron realizar esta actividad en el cuestionario anónimo de partida, tan sólo siete (la mayoría chicas con más de 5 años de residencia en Cataluña) indicaron específicamente disfrutar contactando con familiares/amigos que permanecen en el país de nacimiento y algunos mencionaron la dificultad de establecer esta comunicación de carácter transnacional por las diferencias horarias vigentes. Pero todavía fue más reducido el número de jóvenes que enfatizaron la idea de poder hacer amigos de cualquier punto del mundo a través de la WWW, apenas dos casos.

Chatear por el msn. Por que asi no me aburro en casa i puedo bablar con mis compañeros y amigos. $Y$ de vez. en cuando con algún amigo de mi país (Chica, Bolivia, 17(2) años).

Me gusta conectarme al msn, porque hablo con mis amigos que están en Argentina y con mi familia (Chica, Argentina, 16(7) años).

Con mucha gente... de todos los lados, hasta, hasta Hungria Si. Si... Estuve hablando con un chico que es de Irán y... y luego también españoles... de todos los sitios (Chica, Argentina, 19(6) años).

Otra actividad que me gusta es estar en Internet chateando con mis amigos y, sobre todo, con mi familia que está muy lejos de aqui (Chica, Ecuador, 17 (6) años).

Las "redes sociales" más citadas fueron Facebook, Hi5, Fotolog, Tuenti y MySpace. Sobre el tema de la seguridad, cuando se usa este tipo de servicios, parece que, en términos generales, los padres no realizan ningún tipo de supervisión o control, ya que prácticamente todos los jóvenes apuntaron conectarse desde su habitación y no tener ninguna restricción en este sentido. Únicamente los jóvenes que han vivido o conocen a alguien que ha experimentado 
alguna situación peligrosa parecen adoptar medidas de seguridad como, por ejemplo, restringir el acceso a sus perfiles o al material audiovisual que colocan en la red. En lo que se refiere a la organización de encuentros presenciales vía Internet, sólo una joven de Ecuador (17 años) explicó haber asistido a una convocatoria en una céntrica cafetería de la ciudad de Barcelona que había estado planificada desde un forum musical.

Ahora bien, todo esto no quiere decir que las relaciones personales cara a cara estén entrando en crisis. "Estar con los amigos fuera del entorno escolar" es la actividad preferida por la mayoría de los chicos y las chicas consultados. En el caso de ellos, estos encuentros están estrechamente ligados con la práctica de algún deporte (sobre todo, baloncesto y fútbol).

[Me gusta] salir con mis amigas, para no aburrirme en casa, ir de shopping, ir a una fiesta y conocer gente (Chica, Bolivia, 17 (4) años).

Me gusta salir con las amigas porque aunque las vea aqui en el cole no es lo mismo. Porque en la calle podemos hablar más y por que me lo paso bien (Chica, República Dominicana, 16 (5) años).

Me encantan los deportes como: fütbol, tenis, bicicleta, correr o hacer ejercicios y porque me distraigo me desestreso i me olvido de las cosas negativas que me van pasando (Chico, Ecuador 15 (2) años).

\section{Consideraciones finales}

Coincidiendo con el cierre de esta investigación, un joven ecuatoriano de 17 años fue asesinado en una plaza de L'Hospitalet de Llobregat el 18 de septiembre de 2009. Según recogió la prensa en aquel momento, el chico vivía con unos familiares, ya que sus progenitores permanecían en Ecuador, y, por el modo en que se produjo el asesinato, éste parecía premeditado. Fue una noticia triste, conmovedora, dramática y, a nuestro pesar, coherente con la difícil convivencia que había quedado plasmada en los encuentros que habíamos mantenido con los estudiantes latinoamericanos y autóctonos durante nuestro trabajo de campo ${ }^{10}$. Una convivencia marcada por la tendencia a crear grupos endógenos (con un prácticamente nulo grado de multiculturalidad en los grupos de amistades), dándose un contexto en el que el uso de las tecnologías de la comunicación no puede hacer otra cosa que contribuir en ese mismo sentido, hacia la segregación.

David Morley (2005), desde el área de los Estudios Culturales, ha hecho especial hincapié en las dificultades que tiene la población inmigrada a la hora de acceder a la oferta comunicativa disponible y en el posible dominio de un consumo segregacionista de la misma acorde con la tendencia a vivir en comunidades apartadas (guetos). Tras este trabajo de investigación, resulta todavía prematuro ir más allá de las reflexiones que ya se han apuntado junto a la descripción de los datos, pero sí podemos advertir que, en lo referente a la aproximación de los adolescentes latinoamericanos a la WWW y a la telefonía móvil en Cataluña, el acceso a estas tecnologías no resulta problemático y, en cambio, sí resulta preocupante que su uso tienda efectivamente hacia la segregación. Resulta llamativo que una tecnología como ésta, que permite "estar abiertos al mundo", no parezca propiciar procesos de sociabilidad permeables a la creación de grupos de amistades multiculturales; pero, al mismo tiempo, ello es coherente con el tipo de relaciones sociales que los adolescentes mantienen de forma no mediada. Las relaciones mediadas -como no podía ser de otra manerano pueden ir en sentido contrario a las relaciones que se producen de forma presencial. En consecuencia, y de cara a la consolidación de esta línea de investigación, hay que tener en cuenta que el estudio de las relaciones mediadas no puede realizarse al margen o de manera independiente del entorno socio-afectivo general.

De cara a las políticas sociales que pueden definirse para gestionar el fenómeno migratorio, la conclusión es clara. Todavía queda mucho por hacer y esperamos que el paso del tiempo ayude a que la convivencia multicultural se vaya convirtiendo en un hecho normalizado. A raíz de este trabajo, podemos apuntar dos interpretaciones que permiten encarar el futuro con cierta esperanza. Por un lado, los autóctonos prefieren reunirse con otros autóctonos en su tiempo libre, pero también hay que tener en cuenta que estas amistades, formadas en su mayoría durante la infancia, se produjeron en un momento en el que el volumen de población inmigrada era prácticamente irrelevante. Por su parte, los migrantes optan básicamente

${ }^{10}$ Es más, en los foros abiertos en algunos medios digitales, la noticia provocó una larga lista de mensajes racistas y xenófobos. 
por aproximarse a personas que también han vivido la experiencia que supone emigrar del país de nacimiento, pero hay que advertir que mayoritariamente apuestan por conocer a nuevas personas en el nuevo entorno de convivencia (de forma paralela o no al mantenimiento de contactos transnacionales). Quizá el momento clave está en la tercera generación, tal y como da a entender Kymlicka (1996).

\section{Referencias}

AGUADO, J.M.; MARTINEZ, I.J. 2006. E1 proceso de mediatización de la telefonía móvil: de la interacción al consumo cultural. Zer, 11(20):319-343.

CASTELLS, M. 2006. Comunicación móvil y sociedad: una perspectiva global. Barcelona, Ariel, 400 p.

COGO,D.; GUTIÉRREZ, M.; HUERTAS, A. 2008. Migraciones transnacionales y medios de comunicación. Madrid, Catarata, $164 \mathrm{p}$.

COLEMAN, J.C. 1985. Psicología de la adolescencia. Madrid, Morata, $292 \mathrm{p}$.

HANNERZ, U.1998. Conexiones transnacionales: cultura, gente, lugares. Madrid, Cátedra, 290 p.
IGARZA, R. 2009. Burbujas de ocio: nuevas formas de consumo cultural. Argentina, La Crujía, 245 p.

KYMLICKA, W. 1996. Ciudadanía multicultural. Barcelona, Paidós, 303 p.

LÉVY, P. 2007. Cibercultura: la cultura de la sociedad digital. Barcelona, Anthropos Editorial, 230 p.

LIVINGSTONE, S. 1998. Mediated Childhoods: A comparative approach to young people's changing media environment in Europe. European Journal of Communication, London, 13(4):435456. http://dx.doi.org/10.1177/0267323198013004001

MERTON, R.K.1957. Social stratification: A comparative analysis of structure and process. Nueva York, Harcourt Brace, 540 p.

MORLEY,D.2005. Pertenencias: lugar, espacio e identidad en un mundo mediatizado. In: L. ARFUCH (compil.), Pensar este tiempo: espacios, afectos, pertenencias. Buenos Aires, Paidós, p. 129-168. PRADA, M.A. 2005. ¿'Invención' de la adolescencia migrante? In: Congreso Ser Adolescente hoy, Madrid, 2005. Accesado en: 16/12/2009, disponible en: http://www.fad.es/sala_lectura/ CSAHoy_MR2.pdf.

SCOLARI, C. 2008. Hipermediaciones: elementos para una teoría de la Comunicación Digital Interactiva. Barcelona, Gedisa, 317 p. ZANFRINI, L. 2007. La convivencia interétnica. Madrid, Alianza Editorial, 184 p.

Submetido em: 16/12/2009 Aceito em: 03/02/2010 